\title{
Comparative study between uses of GnRH- agonist versus hCG as an ovulation trigger in patients with polycystic ovary syndrome in antagonist protocol
}

\author{
Poornima Nadkarni, Kishor Nadkarni, Pooja Nadkarni, Prabhakar Singh, \\ Mandeep Bhandal, Aditi Iche*
}

21 Century Hospitals Pvt. Ltd. \& Test Tube Baby centre, Station Rd, Surat, Gujarat, India

Received: 18 June 2015

Revised: 21 June 2015

Accepted: 10 July 2015

\section{*Correspondence:}

Dr. Aditi H. Iche,

E-mail: aditi.iche@gmail.com

Copyright: ( $)$ the author(s), publisher and licensee Medip Academy. This is an open-access article distributed under the terms of the Creative Commons Attribution Non-Commercial License, which permits unrestricted non-commercial use, distribution, and reproduction in any medium, provided the original work is properly cited.

\begin{abstract}
Background: Polycystic ovary syndrome is one of the major causes of infertility. Almost $75 \%$ of ovulatory women have PCOS. Ovarian hyperstimulation syndrome and multiple pregnancies are known complications of PCOS in ART. Many studies are available now, to reduce the incidence and severity of OHSS in these patients, at the same time achieving acceptable pregnancy rate .In our study, we used Antagonist protocol in PCOS patients and compared the results using GnRH-Agonist versus hCG as ovulation trigger.

Methods: This is double blinded comparative study between uses of GnRH- Agonist versus hCG as an ovulation trigger in 100 patients with polycystic ovary syndrome in antagonist protocol, done in private ART setting. In the study, 100 patients randomly allotted in two groups (A \& B), each 50 patients, given ovulation trigger (When leading three follicles were $>18 \mathrm{~mm}$ ) as group A-GnRH-Agonist (Inj. Triptoreline $0.1 \mathrm{mg}, 12$ hours apart two doses subcutaneously) and group B hCG as (Inj. Recombinant hCG, $250 \mathrm{mcg}$ single dose subcutaneously).

Results: In our study, In 50 patients of group A, total 31 patients were pregnant. In group B of 50 cases, 29 patients were pregnant. There was no significant difference between two groups $(\mathrm{P}>0.05)$. Incidence of ovarian hyperstimulation syndrome is significantly less in PCOS patients, when GnRH agonist is used as ovulation trigger in Antagonist protocol, as compared to hCG $(\mathrm{P}<0.01)$. The incidence was mild OHSS was significantly $(\mathrm{P}<0.05)$ less in GnRH group. There was no significant difference in incidence of moderate OHSS $(\mathrm{P}>0.05)$. None patient of two groups developed severe OHSS.

Conclusions: Incidence of the ovarian hyperstimulation syndrome is significantly less in patients with Polycystic Ovarian Syndrome when GnRH agonist is used as an ovulation trigger, as compared to hCG, in Antagonist protocol. In our study, there was no significant difference in pregnancy rates between two groups.
\end{abstract}

Keywords: Polycystic ovary syndrome, Anovulatory, Hyperstimulation

\section{INTRODUCTION}

The introduction of GnRH agonist as a trigger combined with gonadotropins in antagonist protocol can be considered as a major way to prevent OHSS in high risk patients. Polycystic ovary syndrome is one of the major causes of infertility. Almost $75 \%$ of an ovulatory woman have PCOS. ${ }^{1}$ Ovarian hyper stimulation syndrome and multiple pregnancy are known complications of PCOS in ART.

Many studies are available now to reduce the incidence and severity of OHSS in these patients at the same time achieving acceptable pregnancy rate. In our study, we used antagonist protocol in PCOS patients and compared 
the results using GnRH-Agonist versus hCG as ovulation trigger. The doses of GnRH agonist to trigger ovulation vary from 0.1 to $0.5 \mathrm{mg}$ either single or multiple doses 8 , 12 or 24 hours apart .We used it as two doses $0.1 \mathrm{mg}$ twelve hours apart.

\section{METHODS}

This is double blinded Comparative study between uses of GnRH- Agonist versus hCG as an ovulation trigger in 100 patients with polycystic ovary syndrome in Antagonist protocol, done in private ART setting.

In the study, 100 patients randomly allotted in two groups (A \& B), each 50 patients, given ovulation trigger (When leading three follicles were $>18 \mathrm{~mm}$ ) as

A. GnRH-Agonist( Inj. Triptoreline $0.1 \mathrm{mg}, 12$ hours apart two doses subcutaneously) or

B. hCG as (Inj. Recombinant hCG $250 \mathrm{mcg}$ single dose subcutaneously)

- $\quad$ OPU done after 36 hours of trigger.

- $\quad$ ICSI done by embryologist in the same lab and settings.

- $\quad$ Patient underwent a single day 3 transfer of grade one embryo.

- Patients advised luteal phase support (vaginal micronized progesterone tab 400 mg HS, vaginal progesterone Gel $(8 \%)$.

- Serum bhCG done after 12 days and positive cases advised transvaginal ultrasonography on day 35 .

\section{Study parameters:-}

1. Total numbers of oocytes retrieved

2. Total numbers of M2 oocytes

3. Total number of grade one embryos(Morphology grading)

4. Pregnancy rate

5. Incidence of OHSS

\section{Inclusive criteria:-}

1. PCOS patients were diagnosed on basis of Rotterdam Criteria( two of the following) :

- Oligo-and/or anovulation

- Clinical and /or biochemical signs of hyperandrogenism and

- Polycystic ovary on ultrasound scan, defined as the presence of 12 or more follicles in each ovary (one ovary being sufficient for diagnosis)measuring 2-9 $\mathrm{mm}$ in diameter, and/or ovarian volume $>10 \mathrm{~mm}$

2. Husband having normal semen analysis.

3. Patients stimulated with Antagonist protocol.

4. Patients given ovulation trigger with Agonist or hCG.
5. Patients started with progesterone support on day of OPU (Vaginal progesterone $400 \mathrm{HS}$ ).

6. ICSI pregnancy.

7. Patients with single day 3 transfer of grade one embryo.

8. Patients given luteal phase support (vaginal progesterone tab $400 \mathrm{mg}$ HS, vaginal progesterone Gel.

\section{Exclusive criteria:-}

1. Non PCOS

2. Husband having abnormal semen analysis.

3. Dual trigger

4. Lost follow up

\section{RESULTS}

The Table- 1 is showing that, average numbers of oocytes retrieved were 24 in group A, of which 19 (79.1\%) were in M2 phase.

Table 1: Parameters in group A.

Parameters GnRH Agonist

\begin{tabular}{|ll|}
\hline Average numbers of oocytes retrieved & 24 \\
\hline Average numbers of M2 oocytes & $19(79.1 \%)$ \\
\hline
\end{tabular}

The Table-2 is showing that, average numbers of oocytes retrieved were 26 in group B, of which $21(80.7 \%)$ were in M2 phase.

Table 2: Parameters in group $B$.

\begin{tabular}{|ll|}
\hline Parameters & hCG \\
\hline Average numbers of oocytes retrieved & 26 \\
\hline Average numbers of M2 oocytes & $21(80.7 \%)$ \\
\hline
\end{tabular}

Following Table no. 3 showing that, there was no difference in number of grade one embryo formation in both groups.

Table 3: Average number of grade one embryos in group $A$ and $B$.

\begin{tabular}{|ll}
\hline Average number of grade one embryos & Total \\
\hline GnRH Agonist ( Group A) & 4 \\
\hline hCG (Group B) & 5 \\
\hline
\end{tabular}

Following Table 4 shows that, there is no proven difference in group A and group B.

Table 4: Pregnancy rate in group $A$ and $B$.

\begin{tabular}{|ccc|}
\hline Pregnancy rate & Total & $\%$ \\
\hline GnRH Agonist(Group A) & $31 / 50$ & $62 \%$ \\
\hline hCG(Group B) & $29 / 50$ & $58 \%$ \\
\hline
\end{tabular}


Following Table 5 shows that, there is no statically proven difference in group $\mathrm{A}$ and $\mathrm{B}$.

Table 5: Comparison between Pregnancy rate in group $A$ and $B$.

\begin{tabular}{|c|c|c|c|c|}
\hline Parameter & $\begin{array}{l}\text { Group } \\
A(n=50)\end{array}$ & $\begin{array}{l}\text { Group B } \\
(\mathrm{n}=50)\end{array}$ & $\begin{array}{l}\mathrm{Z} \\
\text { Value }\end{array}$ & $\begin{array}{l}\text { P } \\
\text { Value }\end{array}$ \\
\hline $\begin{array}{l}\text { Pregnancy } \\
\text { rate }\end{array}$ & $31(62)$ & $29(58)$ & 0.41 & $>0.05$ \\
\hline
\end{tabular}

Following Table 6 shows the incidence of OHSS in group A which is of three types mild, moderate and severe.

Table 6: Incidence of OHSS in group A.

\begin{tabular}{|lll|}
\hline Incidence of OHSS & GnRH Agonist & $\%$ \\
\hline Mild & 2 & $4 \%$ \\
\hline Moderate & 1 & $2 \%$ \\
\hline Severe & 0 & $0 \%$ \\
\hline
\end{tabular}

Following table 7 shows the incidence of OHSS in group $\mathrm{B}$ which is of three types mild, moderate and severe.

Table 7: Incidence of OHSS in group $B$.

\begin{tabular}{|lll|}
\hline Incidence of OHSS & hCG & $\%$ \\
\hline Mild & 9 & $18 \%$ \\
\hline Moderate & 3 & $6 \%$ \\
\hline Severe & 0 & $0 \%$ \\
\hline
\end{tabular}

Following table 8 shows the comparison of incidence of OHSS in group A and group B. Incidence of ovarian hyperstimulation syndrome is significantly less in PCOS patients, when GnRH agonist is used as ovulation trigger.

Table 8: Comparison of incidence of OHSS in group $A$ and $B$.

\begin{tabular}{|lllll|}
$\begin{array}{l}\text { OHSS } \\
\text { incidence }\end{array}$ & $\begin{array}{l}\text { Group A } \\
(\mathrm{n}=50)\end{array}$ & $\begin{array}{l}\text { Group B } \\
(\mathrm{n}=50)\end{array}$ & $\begin{array}{l}\mathrm{Z} \\
\text { Value }\end{array}$ & $\begin{array}{l}\text { Palue } \\
\text { Mild }\end{array}$ \\
$2(4)$ & $9(18)$ & 2.29 & $<0.05$ \\
\hline Moderate & $1(2)$ & $3(6)$ & 1.03 & $>0.05$ \\
\hline Overall & $3(6)$ & $12(24)$ & 2.60 & $<0.01$ \\
\hline
\end{tabular}

\section{DISCUSSION}

In normal cycle mid-cycle LH surge initiates maturation of oocyte which eventually results in the ovulation. The normal LH surge has duration of approximately 48 hours. $^{2}$ The normal LH surge can be divided into three phases, ascending limb (14 hours), a plateau phase (14 hours), long descending phase (20 hours). GnRH agonist gives same amplitude of LH surge as seen in normal menstrual phase consisting of two phases: a short ascending phase (> 4 hours) and descending phase $(>20$ hrs). Following are the explanations of advantage of GnRH agonist over hCG:-
- $\quad$ GnRH agonist has shorter half-life (60 min) than Hcg (> 24 hours). ${ }^{3}$ The induction of more physiologic surge of ovulatory LH and FSH with GnRH agonist.

- This time-limited stimulus can be restricted to few follicles that are more mature thus lowering number of corpus lutea and OHSS.This is observed that hCG has sustained luteotrophic effect and supraphysiological levels of estradiol and progesterone having negative impact on endometrial receptivity. ${ }^{4,5}$

In our study, in 50 patients of group A, total 31 patients were pregnant. In group B of 50 cases, 29 patients were pregnant. There was no significant difference between two groups $(\mathrm{P}>0.05)$. Incidence of ovarian hyperstimulation syndrome is significantly less in PCOS patients, when GnRH agonist is used as ovulation trigger in Antagonist protocol, as compared to hCG $(\mathrm{P}<0.01)$. The incidence was mild OHSS was significantly $(\mathrm{P}<0.05)$ less in GnRH group. There was no significant difference in incidence of moderate OHSS ( $\mathrm{P}>0.05)$. None patient of two groups developed severe OHSS.

There was some evidence that using GnRH agonist trigger ovulation reduces the incidence of OHSS but needed luteal support. As stated in 2011, GnRHa triggering is a valid alternative to $\mathrm{hCG}$ triggering, resulting in an elimination of OHSS. After modified luteal support there is now a non-significant difference of $6 \%$ in delivery rate in favour of hCG triggering and (Kol.2004) reported that luteolysis induced by GnRH agonist prevent OHSS. ${ }^{6,7}$ Same results were presented previously also as, the GnRH-a appears to be an effective alternative to hCG for inducing the follicular rupture in stimulated cycles in women who are at risk for developing ovarian hyperstimulation syndrome. However, GnRH- an administration can induce short luteal phase. This defect may be ascribed to the pituitary desensitization rather than to a direct effect on corpus luteum. Luteal phase support is needed to prevent luteal phase deficiency. ${ }^{8}$ We provided luteal support in the form of vaginal micronized progesterone tablets and gel.

Itzkovitz et al. reported that $\mathrm{GnRH}$ agonist triptoreline $0.2 \mathrm{mg}$ effectively triggered an endogenous LH surge for final oocyte maturation after garnirelix treatment in stimulated cycles and it prevented OHSS. In high responders with more than 21 follicles and high serum E2 levels $(3000 \mathrm{pg} / \mathrm{ml}), 83 \%$ oocytes were metaphase two and none developed OHSS. ${ }^{9}$

Lewit et al. reported the outcome of 73 treatment cycles involving 44 high responders with previous history of OHSS and high estradiol levels $(>13,200 \mathrm{pmol} / \mathrm{L})$ on the day of triggering using GnRH agonist. None developed of ovarian hyper stimulation syndrome, But required luteal support. ${ }^{10}$

Dong et al. reported that using GnRH agonist to trigger oocyte maturation and ovulation in PCOS patients using gonadotropins with raised E2 levels. 83.3 percent ovulated and $22.2 \%$ became pregnant, only one of the 18 cycles ended in OHSS. ${ }^{11}$ Tay in 2002 stated that GnRH agonist is able to induce an endogenous surge of LH and 
FSH which is physiological and prevent incidence of OHSS. $^{12}$

In 1994 Shaler et al. also stated that GnRH agonist reduces the incidence of OHSS. They reported 6 out of 12 got pregnant and none of twelve developed OHSS. ${ }^{13}$ The mean number of oocytes, proportion of mature oocytes and fertilization rate were similar between the study and control groups. Implantation rate $(38.6 \%$ versus $45.1 \%)$, clinical pregnancy rate $(69.6 \%$ versus $60.9 \%)$ and delivery rate $(62.5 \%$ versus $56.5 \%)$ were similar in the study and control groups respectively. There was one case of moderate ovarian hyperstimulation syndrome (OHSS) in the control group and none in the study group. GnRH agonist is effective in triggering oocyte maturation in patients with PCOS or previous high response. $^{14}$

Ovarian hyperstimulation mainly causes increased vascular permeability, VEGF known to be major factor for the same. In one study, in patients with PCO treated with FSH/ GnRH antagonist, final oocyte maturation with GnRH agonist instead of HCG reduces significantly inhibit A, E2 and progesterone levels during the luteal phase. This phenomenon reflects the inhibition of the corpus luteum function and may explain, at least in part, the mechanism of OHSS prevention in high-risk patients but the study do not support a crucial role for VEGF or TNF $\alpha$ in OHSS. ${ }^{15}$

In our study we concluded that, incidence of the ovarian hyperstimulation syndrome is significantly less in patients with Polycystic Ovarian Syndrome when GnRH agonist is used as an ovulation trigger, as compared to hCG, in antagonist protocol. In our study, there was no significant difference in oocytes retrieved, number of grade one embryo and pregnancy rates between two groups. We provided luteal support in the form of vaginal micronized tablets and gel.

\section{Funding: No funding sources}

Conflict of interest: None declared

Ethical approval: Approved by ethics committee

\section{REFERENCES}

1. Gorry A, White DM, Franks S. Infertility in Polycystic Ovary Syndrome. Endocrine. 2006;30(1):27-33 .

2. Hoff JD, Quigley ME, Yen SSC. Hormonal Dynamics at mid-cycle: a re-evaluation. J Clin Endocrinol Metab 1983;57:792-96

3. Yen SSC, Llenera G, Little B, Pearson OH. Disappearance rate of endogenous luteinisation hormone and chorionic gonadotropin in man. J Clin Endocrinol Meatab 1968;28:1763-967.

4. Forman R, Fries N, Testart J, Belaiseh J, Hazout A, Frydman R. Evidence of an adverse effect of elevated serum estradiol concentrations on embryo implantation. Fertil Steril 1988;49:118-22.
5. Simin C, Garcia Velasco JJ, Valbuena D, Peinado JA, Moreno C, Remohi J, Pellicer C. Increasing uterine receptivity by decreasing estradiol levels during the preimplantation period in high responders with follicle stimulating hormone step- down regimen, Fertil Steril.1998;70:234-39.

6. P. Humaidan, S. Kol, E.G. Papanikolaou. GnRH agonist for triggering of final oocyte maturation: time for a change of practice? Hum Reprod Update, 17 (2011), pp. 510-524.

7. Kol SL. Luteolysis induced by a gonadotropins releasing hormone agonist is the key to prevention of ovarian hyper stimulation syndrome. Fertil Steril.2004,81:1-5.

8. A. Lanzone, A.M. Fulghesu, P. Villa, C. Guida, M. Guido, M.C. Nicoletti et al. Gonadotropin-releasing hormone agonist versus human chorionic gonadotropin as a trigger of ovulation in polycystic ovarian disease gonadotropin hyperstimulated cycles, Fertil Steril, 62 (1994), pp.35-41.

9. Itskovitz-Eldor J. Kol S, Mannaerts B. Use of single bolus of $\mathrm{GnRH}$ agonist triptorelin to trigger ovulation after GnRH antagonist ganirelix treatment in women undergoing ovarian stimulation for assisted reproduction, with special reference to prevent ovarian hyper stimulation syndrome ,Human reprod 2000:15(9).

10. Lewit N, Kol S, Manor D. Itskovitz -Eldor J. The use of GnRH analogue for induction of the preovulatory gonadotropin surge in assisted reproduction and prevention of ovarian hyper stimulation syndrome. Gynecol Endocrinol. 1995;9(suppl 4):13-17.

11. Dong H. Chen S, Xing F. Application of gonadotropins releasing hormone agonist for triggering ovulation in high risk gonadotropin stimulating cycle in infertile polycystic ovarian syndrome patients. Zhonghua Fu Chan Ke Za Zhi. 1999;34(2):94-96.

12. Tay CC. Use of gonadotropins releasing hormone agonists to trigger ovulation, Hum Fertil (camb). 2002;5(1):G 35-37.

13. Shalev E, Geslevich Y and Ben-Amc. Induction of preovulatory luteinizing hormone surge by gonadotropins releasing hormone agonist in patients with high risk of ovarian hyper stimulation syndrome Hu. Reprod.1994;9:417-9.

14. L Engmann, L Siano, D Schmidt, J Nulsen. GnRH agonist to induce oocyte maturation during IVF in patients at high risk of OHSS, Vol. 13,Issue 5,2006 page no. 639-644 Reprductive Biomedicine.

15. Babayof PR, Margalioth EJ, Huleihel M, Amash A, Zylber-Haran E, Gal M, et al. Serum inhibin A, VEGF and TNF alpha levels after triggering oocyte maturation with GnRH agonist compared with HCG in women with polycystic ovaries undergoing IVF treatment: a prospective randomized trial. Hum Reprod. 2006;21:1260-1265.

Cite this article as: Nadkarni $\mathrm{P}$, Nadkarni $\mathrm{K}$, Nadkarni P, Singh P, Bhandal M, Iche A.

Comparative study between uses of GnRH- agonist versus hCG as an ovulation trigger in patients with polycystic ovary syndrome in antagonist protocol. Int J Reprod Contracept Obstet Gynecol 2015;4:1161-4. 